\title{
Paraneoplastic scleroderma or neoplasia secondary to scleroderma
}

\author{
Alexandru Arvunescu' ${ }^{1}$, Adelina lanuli-Arvunescu ${ }^{2}$ \\ ${ }^{1}$ Medical Department, "Th. Burghelea" Hospital, Bucharest, Romania \\ ${ }^{2}$ Medical Department, Colentina Hospital, Bucharest, Romania
}

\begin{abstract}
Scleroderma is one of the rare illnesses from the group of autoimmune diseases, with unclear etiology, that starts with skin involvement and leads to systemic complications. As in many other autoimmune diseases, the main part in the pathogenesis is represented by an abnormal activation of the T lymphocytes. Risk factors include: family history, certain genetic factors, silica exposure and the diagnosis is confirmed by immunological tests (anti Scl70 antibodies). Scleroderma patients have a higher risk to develop, throughout their life, a malignancy (lung, breast, hematologic and urinary bladder type).

We are discussing the case of a 55 year male patient, diagnosed 10 years ago with systemic sclerosis, who suddenly develops a speech disorder - anomic aphasia - as an isolated and sole manifestation. Cranial magnetic resonance imaging (MRI) revealed a mass in the left parieto-temporal area, highly sugestive of metastasis. Whole-body computed tomography (CT) scan could not identify the primary lesion. After surgical resection, the histopatological exam (combined with immune histochemistry $[\mathrm{IHC}]$ and genetic tests) confirmed the metastasis of a lung adenocarcinoma.

Bearing in mind that coexistence of the two diseases may be totally unrelated, this case rises some unsolved questions: is scleroderma a paraneoplasic manifestation or the malignancy is induced by the immunosupressive therapy used to treat scleroderma.
\end{abstract}

Keywords: scleroderma, malignancy, lung cancer

\section{INTRODUCTION}

Scleroderma is one of the rare diseases from the group of collagenoses, with an incidence per year of about 3 new cases per 100,000 people, affecting mostly women (female to male ratio 4:1 or greater). Under the name of scleroderma, there are several types of disease: localized type scleroderma (localized morphea, pansclerotic morphea, linear scleroderma) and systemic type scleroderma (CREST syndrome and progressive systemic sclerosis - the most common) $(1,2,3)$.

The disease usually produces changes in the skin, blood vessels, muscles and internal organs (lung, heart, kidney). The most common characteristic presentation of scleroderma is thickening of the skin which causes stiffness and reduced range of motion of surrounding joints. Vascular involvement produces reduced peripheral blood flow which causes the Raynaud's phenomenon and, in severe cases, acral necrosis. Although the main characteristic of sclero- derma is the involvement of the skin, there is a very particular condition called, sscleroderma sine scleroderma", in which the skin is sparred and only the internal organs are affected.

The cause of the disease is unknown; however, the current explanation of pathogenesis is an abnormal activation of $\mathrm{T}$ lymphocytes, which causes an increase in collagen synthesis, leading to altered connective tissue and sclerosis. Risk factors include family history, certain genetic factors, and exposure to silica $(2,3)$.

Diagnosis is typically based on a person's symptoms and may be supported by a skin biopsy or blood tests detecting antitopoisomerase antibodies: anti Scl70 (specific for systemic sclerosis), or/and anticentromeric antibodies (specific for CREST syndrome) (4).

The major complications that are responsible for death in patients with scleroderma are: pulmonary hypertension, pulmonary fibrosis and the so called 
scleroderma renal crisis. At the same time, people with scleroderma are also at a higher risk for developing several cancers (lung, breast, haematologic and urinary bladder) $(6,7,8,9)$.

\section{CASE REPORT}

We are reporting the case of a 55 year old male, active smoker (40 pack-years), without any professional exposure to noxes and no documented allergy to any substance. He presented to the emergency room after 48 hours of experiencing a sudden speech disorder - anomic aphasia (a particular case of aphasia, in which an individual cannot express the words he wants to say, but otherwise has no problem in building sentences). The patient had neither any previous neurological symptoms, nor any cognitive impairment or any other complaints, whatsoever (headache/vision disturbances/movement or sensitive impairment).

The patient is known to the clinic since 2008, when he was diagnosed with scleroderma after developing a thickening of the skin associated with stiffness and Raynaud's phenomenon. Blood tests showed constant high levels of anti Scl 70 antibody ( $214 \mathrm{UI} / \mathrm{ml})$. His scleroderma is rather constituted, since he already had multiple complications at the moment of the first presentation to the hospital: pulmonary fibrosis, right pachypleuritis, amputation of the $\mathrm{V}$ left toe, secondary to necrosis, calcanean ulceration and deforming scars of the fingertips in both hands. He associates iatrogenous Cushing syndrome, being currently corticodependent, mitral valve prolapse and also metabolic syndrome: grade 2 arterial hypertension, grade 2 obesity, dislipidemia (high cholesterol, LDL-cholesterol and triglycerides) and impaired glucose tolerance.

In the last 10 years he regularly came to his follow-ups. Regarding the pharmacological treatment, he constantly received corticotherapy (methylprednisolone), cyclophospamide (initially 3 courses of pulse therapy, presently on oral therapy), vasodilatators (sulodexide, pentoxifylline and amlodipine), metoprolol and gastric protection (omeprazol). Poor control of acral ulcers, determined the administration of the endothelin inhibitor bosentan, which reduced the number and severity of ulcerations and the time to their healing.

On the current admission, physical examination revealed that the patient was afebrile, with a normal heart rate of 75 beats per minute, blood presure of
$140 / 85 \mathrm{mmHg}$, slightly anxious, but being able to communicate and fulfill certain tasks. The neurological exam didn't yield anything unusual, with the exception of the speech disorder already mentioned. Normal pulmonary sounds were present, bilaterally, with no apparent dullness on percussion and fine crackles in the base of the right hemithorax. Cardiac auscultation unfolded a grade 3 mezo-systolic murmur audible in the mitral area, radiating to the axilar region, with no other extra sounds. There were no signs of central or periphereal cyanosis, and both legs had pulsatile pedal arteries. There was digital necrosis of the fifth left toe (Figure 1). The patient dind't experience any weight loss, pain, or syncope recently.

The ECG showed sinus rhythm with sequelar q waves in DIII and aVF leads.
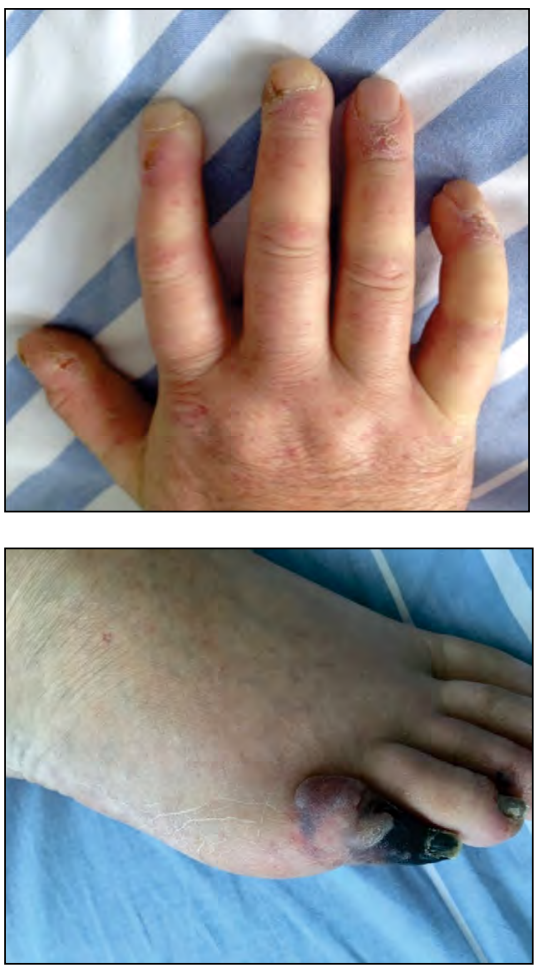

FIGURE 1. The image on the left shows the skin thickening of the right hand; the image on the right shows the acral necrosis of the fifth left toe

Given the rapid onset of the manifestation that dind't vanish over 48 hours and the lack of other neurological signs, associated with biological inflammation (fibrinogen $=760 \mathrm{mg} / \mathrm{dl}$, VSH $=67$ $\mathrm{mm} / \mathrm{h}, \mathrm{CRP}=63 \mathrm{mg} / \mathrm{l}$ ) we decided to perform a cranio-cerebral MRI scanning. The exam revealed a $28 / 30 / 31 \mathrm{~mm}$ expansive image in the left temporo-parietal lobe with periferic digitiform edema, absence of perilesional sulci and impression of left lat- 


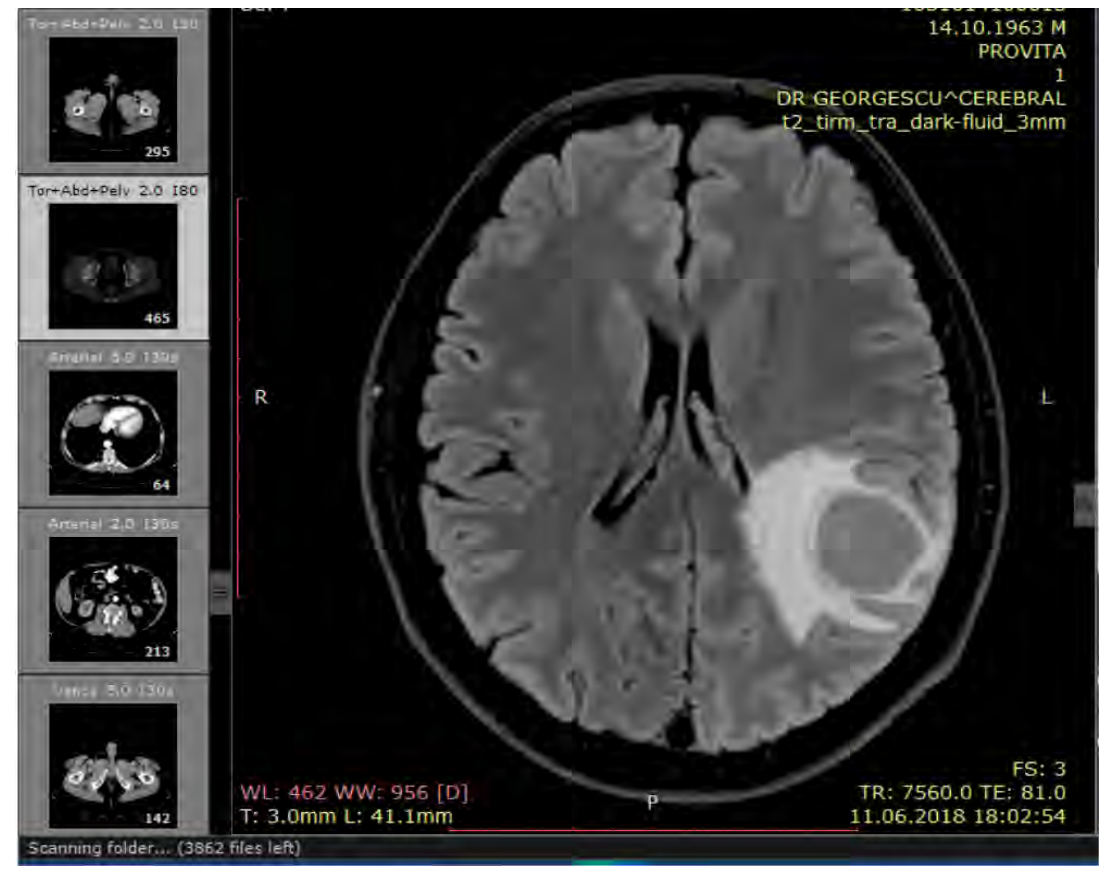

FIGURE 2. MRI scan of the brain showing the image of an expansive lesion in the left temporo-parietal lobe of 28/30/31 mm (antero-posterior, transversal, cranio-caudal) with periferic digitiform edema, absence of perilesional sulci and impression of the left lateral ventricle

eral ventricle. The result was highly sugestive of metastasis (Figure 2).

In order to elucidate the primary tumor site, a whole-body CT scan was performed, which showed multiple subpleural nodules (3-7.4 mm) situated in both lungs (except the inferior right lobe) and right hilum adenopathy $(19 / 15 \mathrm{~mm})$.

The patient was admitted in the neurosurgery department, where an excisional biopsy of the mass was performed. Post-interventional MRI showed complete excision with no residual mass. The histopathological examination including imunohistochemistry and genetic tests confirmed the diagnosis: metastasis of a pulmonary adenocarcinoma with moderate differentiation (EGFR-).

Promptly after surgery, the speech disorder resolved completely.

Shortly thereafter, the patient underwent fractionate stereotactical cranial radiotherapy, which was well tolerated. According to the oncologic protocol, the patient recieved 3 classic chemotherapy courses (Gemcitabine+Carboplatin), with usual side effects (anemia and leucopenia).

The cytostatic treatment was interrupted by the development of a sudden intense pain in the left foot associated with paresthesias. Upon examination, the foot was pale, cold and pulseless. Doppler imaging confirmed the clinical impression of acute arterial obstruction of the lower left limb, visualising a popliteal arterial obstruction. He was admitted to the cardiovascular surgery department where continuous intravenous anticoagulant therapy was initiated, but could not restore the blood flow. Because of that evolution, an interventional desobstruction was performed - endarterectomy - which succesfully restored the blood flow of the left limb.

\section{DISCUSSIONS}

This case rises the possibility of an actual correlation between scleroderma and neoplasic disease, which can be analyzed from the following two points of view: either, the autoimmune disease is actually a paraneoplasic manifestation of the cancer, or the immunosupresive therapy used to treat scleroderma, along with the existing pulmonary fibrosis, led to a neoplasia. Nevertheless, there still is the possibility that the two diseases are concomitent but independent $(7,8,9)$.

As it is already very well known, smoking is a risk factor for the occurence of most lung cancers, especially centro-hilar forms. But this is not the case in the adenocarcinoma histological type (9). As a matter of fact, in this case the primary pulmonary site of the adenocarcinoma could not be identified.

In the literature, scleroderma is considered one of the many paraneoplasic manifestation, that can hide 
a future neoplasic disease. Statistical data from a meta-analysis showed that in the first to 12 moths from the diagnosis of scleroderma the risk of discovering a neoplasia is two times higher than after the first year (OR: 2.79; 95\% CI 1.81, 4.31 vs OR: 1.40; $95 \%$ CI $1.22,1.61 ; \mathrm{p}=0.003$ for comparison) (10). The risk decreases exponentially with every year aded from the diagnosis, being approximately equal to the general population after 3 years. The same meta-analysis observed that $55 \%$ of paraneoplasic scleroderma patients had an elevated level of anti-RNA polimerase III antibody, which the authors consider a predictor. In this case, the long term evolution of the disease (10 years) makes it at least improbable to be a paraneoplasic syndrome.

It is considered by many authors that scleroderma is associated with a higher risk of neoplasia, with the primary sites being lung, breast and cervix cancer, lymphoma, and multiple mieloma. The data collected in a meta-analysis published in 2016 (12) show a variable incidence of neoplasia among scleroderma patients, but all indicated a higher general risk in males (OR: 2.2; 95\% CI: 1.7-2.8) compared with females (OR: 1.3; 95\% CI: 1.1-1.6). However, regarding lung cancer the data are reversed, with a higher risk in females (OR: 5.6 vs. 2.6). The risk was even higher if the patient had high levels of anti Scl70 antibodies (OR: 6.4). The highest risk was in patients that had pulmonary fibrosis with reduced vital capacity on spirometry $(<70 \%)$, OR: 6.7 . The studies were conducted in various parts of the globe: Italy, Taiwan, Sweden, Australia and US (Detroit), a fact that offers to the analysis a more realistic image upon the general perspective, the cohorts in these cases being heterogenous. All the results demonstrated a high risk of developing a lung cancer in scleroderma patients with odd ratios between 3 to 6 , only one showing an OR of 1 ; in this case the explanation was the already high basal risk of the studied population to develop a lung cancer.

Most probably, in this case, given the long time since the diagnosis, and the fact that there are many other patients receiving long term chronic immunosupresive therapy, even in higher doses than our pa- tient, we cannot establish any objective link or direct correlation between these two independent diseases.

After the first 3 cures of chemotherapy, the patient had an episode of acute peripheral ischemia, which required interventional de-obstruction by endarterectomy. The question is which of the following factors caused the ischemia: the proinflammatory status of the scleroderma, the smoking history, the metabolic syndrome associated with a high atherosclerotic risk or the athero-thrombotic status associated with neoplasia. Despite the fact that there are no studies comparing head-to-head the risk of the factors above, there are some data which can offer an answer.

One study in the UK showed a lower risk of ischemia leading to necrosis in non-smoking patients with high levels of anti-centromer antibodies (OR: $3.1)$ in comparison to the active smoking patients (OR: 6.2) (6).

Another paper focused on finding the prevalence of athero-thrombotic events occuring in patients diagnosed with neoplasia (11). The general prevalence was $15 \%$, with the highest in pancreatic cancer $(28 \%)$, followed closely by lung cancers $(27 \%)$.

It is difficult to say which was the major factor that caused the ischemia, but probably all of them played a part in the whole big picture.

\section{CONCLUSIONS}

The scleroderma diagnosis is usually hard to confirm, until anti-topoisomerase specific antibodies are positive in blood tests, because the signs can be overlooked by the examination in the early stages or can be confused with signs of other, more frequent rheumatologic autoimmune diseases. However, taking into consideration the severe invalidant complications and the possible benefits of early diagnosis and treatment, clinicians should have it in mind.

Nevertheless, as in this case, two severe systemic chronic diseases can co-exist independently. Despite lack of correlation, both act simultaneously, leading to possible fatal complications. Perhaps future studies will offer missing pieces to this unsolved puzzle and answer to some of these questions.

\section{REFERENCES}

1. Longo D, Fauci A, Kasper D, Hauser S, Jameson J, Loscalzo J. Harrison's Principles of Internal Medicine (18 ed.). New York: McGraw-Hill Professional, 2011. ISBN 978-0-07174889-6.

2. Barnes J, Mayes MD. Epidemiology of systemic sclerosis: Incidence, prevalence, survival, risk factors, malignancy, and environmental triggers. Current Opinion in Rheumatology 2012. 24 (2): 165-70. 
3. Scleroderma. NORD (National Organization for Rare Disorders). 2007. Archived from the original on 8 September 2016. Retrieved 14 July2017.

4. Jimenez SA, Cronin PM, Koenig AS, O'Brien MS, Castro SV, Varga J, Talavera F, Goldberg E, Mechaber AJ, Diamond HS, eds. Scleroderma Differential Diagnoses. Medscape Reference. WebMD. Archived from the original on 6 March 2014. Retrieved 6 March 2014

5. Valančienė G, Jasaitienè D, Valiukevičienè S. Pathogenesis and treatment modalities of localized scleroderma. Medicina 2010. 46 (10): 649-56.

6. Alenghat FJ. The Prevalence of Atherosclerosis in Those with Inflammatory Connective Tissue Disease by Race, Age, and Traditional Risk Factors. Scientific Reports 2016. 6: 20303.

7. Shah AA, Rosen A. Cancer and systemic sclerosis: Novel insights into pathogenesis and clinical implications. Current Opinion in Rheumatology 2011. 23 (6): 530-5.
8. Szekanecz É, Szamosi S, Horváth Á, Németh Á, Juhász B, Szántó J, Szücs G, Szekanecz Z. Malignancies associated with systemic sclerosis. Autoimmunity Reviews 2012. 11 (12): 852-5.

9. Bonifazi M, Tramacere I, Pomponio G, Gabrielli B, Avvedimento EV, La Vecchia C, Negri E, Gabrielli A. Systemic sclerosis (scleroderma) and cancer risk: Systematic review and metaanalysis of observational studies. Rheumatology 2013. 52 (1): 143-54.

10. Ami A. Shah, Livia Casciola Rosen. Cancer and Scleroderma: A Paraneoplastic Disease with Implications for Malignancy Screening. Curr Opin Rheumatol. 2015 Nov; 27(6): 563-570.

11. Graham J Caine, Paul S Stonelake, Gregory YH Lip, Sean T Kehoe.The Hypercoagulable State of Malignancy: Pathogenesis and Current Debate. Neoplasia. 2002 Nov; 4(6): 465-473.

12. Nabil Zeineddine, Lara El Khoury, Joseph Mosaka. Systemic Sclerosis and Malignancy: A Review of Current Data. J Clin Med Res. 2016 Sep; 8(9): 625-632. 